\title{
Controlling the Degradation of Wireless Sensor Networks
}

\author{
Alba Rozas, Javier Blesa, Elena Romero and Alvaro Araujo
}

\begin{abstract}
With the fast expansion of Wireless Sensor Networks (WSNs) and the increasing emergence of new scenarios and applications, extending their lifetime is crucial. Usually, WSN developers use generic algorithms and deployment arrangements without considering the specific needs of their network's application. Taking this application into account can result in a significant enhancement of performance, both in terms of increasing the lifetime and improving the quality of service (QoS). Furthermore, most WSN developers do not consider the final behavior of the network when nodes are nearly depleted and resources are scarce. In this paper we introduce the concept of the controlled degradation of the network, to refer to the strategies aimed at managing this deterioration process. The existing definitions of the network lifetime do not normally consider the specific purpose or application for which the WSN is intended. Thus, they are not suited to describe and test controlled degradation strategies. Consequently, we propose a new formal and comprehensive definition for the network lifetime. Finally, this work presents a proof of concept that confirms our statements and reinforces the potential of this research line.
\end{abstract}

Keywords - Wireless sensor networks; energy efficiency; network lifetime; controlled degradation

\section{INTRODUCTION}

Wireless communications are continuously expanding and permeating every environment these days. WSNs are one of the fastest growing sectors in the field of wireless and mobile communications. As stated in [1], the number of wireless connected devices exceeded the world's population in 2014 , and by 2019 there will be more than 11.5 billion mobile-connected devices including Machine-to-Machine (M2M) modules.

Nodes in WSNs are generally powered from a limited energy source such as a small battery. Also, they are usually not easily accessible after their deployment. As a result, it is very difficult and undesirable to change their batteries. Given this limited power source, making an efficient use of the energy is a crucial matter in these kinds of networks [2].

Related to the topic of energy efficiency in WSNs emerges the term "network lifetime". Although the concept is widely used by researchers, there is no consensus on its exact definition. Roughly, it is defined as the period in which the network is operational. This notion of operation is not objective, and it strongly depends on the purpose or application for which the network is intended [3].

Taking the network's purpose into account can be very beneficial. This way, algorithms can be specifically applied to reducing consumption and increasing the network lifetime for each individual WSN. Different WSN scenarios may have different requirements so it would not be appropriate to measure and compare their lifetime against a unique definition.

Not only is it interesting to increase their lifetime, but there are also many WSN applications in which it would be very desirable for the user to have control in the process of degradation that comes at the end of the network's operation. In a multiservice network, the degradation can be controlled by discarding some packets, attending to the different services' priorities, when nodes are near exhaustion. As a result, the most important services can be maintained for a longer period at the expense of reducing the lifespan of some low-priority functionalities.

The abovementioned issue leads us to introduce the concept of the controlled degradation of the network. It encompasses all the algorithms or methods aimed at maintaining control about how a certain network will stop functioning. The concept of controlled degradation is intrinsically linked to the specific application or purpose of the WSN. Moreover, it makes it mandatory to include this application in the definition of the network lifetime, as we propose in this paper.

The rest of this paper is organized as follows. In Section II some related works are reviewed. In Section III the concept of controlled degradation is discussed and our proposed definition for the network lifetime is introduced. Section IV shows a proof of concept of these ideas. Finally, some conclusions are shown in Section V.

\section{RELATED WORK}

The concept of controlling the degradation of a network has been widely discussed in the computer science field. It is mostly referred to by the name of "graceful degradation", 
and involves techniques to achieve a smooth cessation of operations in all kinds of scenarios [4].

However, the concept has been rarely applied to WSNs. As an example, the work in [5] presents a congestion control method for wireless communication networks to avoid a sudden degradation of QoS.

There are no works that specifically try to control the degradation process in order to improve the network lifetime, as we propose in this paper. The degradation control concept that we present is intrinsically related to the specific purpose of the WSN so it is necessary to work with a lifetime definition that takes it into account.

There are a lot of different definitions of the network lifetime in the works of this field. In most cases they are related to the appearance of a loss of connectivity in the network. Roughly, the different definitions can be classified into three groups: those related to the time till a percentage of the nodes fail; the time until the appearance of the first partition in the network; and the time till the packet rate drops below a threshold.

Belonging to the first group, a very frequent definition of the network lifetime is the period until the first node of the network depletes its battery, as used by the authors in [6]. This definition is very pessimistic as there are many WSN scenarios in which the network is still fully functional after the failure of a single sensor.

The second group considers the network lifetime to be the time until the appearance of the first partition in the network. A network is considered to have a partition when there is at least a pair of nodes that do not have a path to communicate with each other. The work in [7] uses this kind of definition for the network lifetime. As with the previous type, there are WSN applications in which the appearance of a partition does not imply the end of its operation.

The third group of definitions encompasses those related to the degradation of certain quality of service metrics. The authors in [8] define the lifetime as the time it takes for the packet delivery rate to drop below a certain threshold. Once again, some WSN scenarios may not need a specific quality of service but only certain area coverage.

Some authors have stressed the importance of taking the application into account. For example, the authors in [9] consider that " $[\ldots]$ a good definition of network lifetime should refer to the capability of the network to provide the services it was designed for [...]". In turn, in [7] Dong remarks the fact that "[...] network lifetime should refer to the capability of the network to serve its design purpose".

Finally, Dietrich and Dressler [10] propose a very formal and extensive definition for the network lifetime in WSNs. They have developed a very flexible and comprehensive mathematical model for characterizing the network lifetime. Its main advantage is that it allows for the inclusion of several performance metrics simultaneously. In their work, they also discuss the concept of graceful degradation. The work on this paper is based on their model to a significant extent.

After reviewing the related work of this field, we conclude that none of the existing definitions are completely adequate for describing and testing controlled degradation strategies. In this work we propose a new definition that is comprehensive, flexible and adaptable to different scenarios. It is very well suited to study and characterize the controlled degradation concept because it supports the partial functioning of services.

\section{CONTROLLED DEGRADATION}

As we expressed in the introduction, energy efficient algorithms for WSNs can greatly benefit from considering the network's purpose. This way, not only can algorithms be better suited for each individual WSN scenario, but the desirable controlled degradation of the network can be achieved.

Taking into account the network's purpose implies the need for a flexible and adaptable lifetime definition. It has to be able to handle different performance metrics, suited to the necessities of the different WSN scenarios.

\section{A. Network lifetime definition}

In order to define the network lifetime we need to make use of the concept of network liveliness $\zeta(t)$. It describes the level of functionality of the network and was already introduced in [10]. Our network lifetime $(L)$ will be the period of time from the beginning of its service to the moment its function of liveliness is no longer fulfilled.

The liveliness expression that we propose is not binary so it can reflect a partial level of functionality. As a result, our network lifetime definition will have the network's level of functionality as a parameter. This way, the network lifetime metric can be presented as a time-functionality pairs instead of a single numeric value. This is not a common practice in the works of this field and it could greatly enhance the comparison among algorithms. The following expression is used to represent our network lifetime:

$$
L_{F_{i}}=\left\{t_{i}, F_{i}\right\}
$$

where $t_{i}$ is the time in seconds and $F_{i}$ the level of functionality expressed as a percentage, with $i \in \mathbb{Z}$ representing different possible measuring points.

The following expressions could describe two lifetimes achieved by a certain algorithm in this proposed manner:

$$
\begin{aligned}
& L_{F_{1}}=\left\{t_{1}, F_{1}\right\} \\
& L_{F_{2}}=\left\{t_{2}, F_{2}\right\}
\end{aligned}
$$


In a normal case, if $F_{2}$ is a more reduced functionality than $F_{1}, t_{2}$ would be greater than $t_{1}$.

The network liveliness expression that we propose in this work, $\zeta(t)$, is a time-dependant continuous function that can have values in the range from 0 to 1 . This values represent the level of functionality, $F$, present in the network at a particular time, from 0 (no functionality, $F=$ $0 \%$ ) to 1 (complete functionality, $F=100 \%$ ). The following is its formal definition:

$$
\begin{gathered}
\zeta(t) \in[0,1] \text {, where } \\
\left\{\begin{aligned}
\zeta(t)=0 & \rightarrow \text { No functionality }(0 \%) \\
0<\zeta(t)<1 & \rightarrow \text { Partial functionality }(0 \text { to } 100 \%) \\
\zeta(t)=1 & \rightarrow \text { Complete functionality }(100 \%)
\end{aligned}\right.
\end{gathered}
$$

In addition, the liveliness expression will take the application requirements into account in a dynamic manner. This means that the criteria and performance metrics that contribute to the liveliness expression, and their relative weights, can be dynamically modified during the network's operation. This, combined with the ability to describe partial functionalities is key to our goal of taking the network's purpose into account and being able to control its degradation.

\section{1) Degree of fulfillment of a criterion}

The network liveliness reflects the aggregate degree of fulfillment of several criteria. At this point we need to introduce another expression, $\psi_{* *}$, which is the degree of fulfillment of a certain criterion $* *$. As with the network liveliness, this concept has to be able to describe partial functionalities.

The degree of fulfillment of a certain criterion $* *, \psi_{* *}$, is a time-dependant continuous function such that:

$$
\begin{gathered}
\psi_{* *}(t) \in[0,1], \text { where } \\
\left\{\begin{aligned}
\psi_{* *}(t)=0 & \rightarrow \text { Criterion is not fulfilled at all } \\
0<\psi_{* *}(t)<1 & \rightarrow \text { Criterion is partially fulfilled } \\
\psi_{* *}(t)=1 & \rightarrow \text { Criterion completely fulfilled }
\end{aligned}\right.
\end{gathered}
$$

For most metrics and criteria, the way to achieve the above kind of fulfillment definition is to set two different thresholds, a soft upper bound and a hard lower bound. If the metric is above the soft upper bound, $c_{s u b}$, its corresponding criterion is considered to be completely fulfilled. If it does not surpass the soft upper bound, but it is above the hard lower bound, $c_{h l b}$, the criterion is partially fulfilled. Finally if it does not reach the hard lower bound, the criterion is considered to not be fulfilled at all. This is done without loss of generality, as a different criterion's fulfillment can be described differently, for example by means of a discrete table.

This is better seen with the help of a real example, for instance with the throughput metric. A data collection WSN application may have a certain desired throughput goal. Any throughput above that is considered to provide a perfect functionality and completely fulfill its corresponding criterion. On the other hand, the application may also have a hard threshold, lower than the previous one, that the throughput is absolutely forced to surpass. If this lower bound is not reached, the network is considered non-functional and its collected data would be considered invalid. The first desired soft bound would be $c_{s u b}$ while the latter would be $c_{h l b}$. Any throughput value in between those two bounds is considered to give a partial functionality.

As a result, the degree of fulfillment of the throughput criterion, $\psi_{t h}(t)$, is:

$$
\begin{aligned}
& \psi_{t h}(t)= \\
& \left\{\begin{aligned}
0, & \text { if throughput }<c_{h l b} \\
\frac{\text { throughut }-c_{h l b}}{c_{\text {sub }}-c_{\text {hlb }},} & \text { if } c_{\text {hlb }}<\text { throughput }<c_{\text {sub }} \\
1, & \text { if throughput }>c_{\text {sub }}
\end{aligned}\right.
\end{aligned}
$$

2) Network liveliness

Once we have determined how we are measuring the degree of fulfillment of the different criteria, we are ready to formally derive the liveliness expression, $\zeta(t)$. We want its definition to be able to take into account different criteria, each with a dynamically modifiable weight. As a result, we propose the following liveliness definition:

$$
\zeta(t)=\frac{\sum w_{* *}(t) \cdot \psi_{* *}(t)}{\sum w_{* *}(t)}
$$

where $w_{* *}(t)$ is the weight given to the criterion $* *$, and the sums include all possible criteria.

In order for the liveliness to have values in the continuous range from 0 to 1 , and given our general definition of the level of functionality, the weight functions have the following characteristics. They are timedependant, to reflect the fact that they can dynamically change during the networks operation. Also, they can have continuous values in the range from 0 to 1 , with 0 meaning that the corresponding criterion does not affect at all, and 1 being the maximum possible contribution.

$$
w_{* *}(t) \in[0,1]
$$

\section{3) Network lifetime}

After introducing all of the above concepts, we can now define the network lifetime. The network lifetime for a certain level of functionality $F$, expressed as $L_{F}$, is the period of time from the beginning of the network's operation to the moment the liveliness $\zeta(t)$ stops satisfying that corresponding level of functionality.

The following are some useful examples of this definition. The full lifetime, $L_{100 \%}$, is the lifetime achieved with a $100 \%$ of functionality. It is the period of time until $\zeta(t)$ goes below 1 , which corresponds to the moment when the network stops having a complete functionality. On the other hand, the total lifetime, $L_{0 \%}$, corresponds to the 
TABLE I. NOTATIONS

\begin{tabular}{|c|l|}
\hline Notation & \multicolumn{1}{|c|}{ Meaning } \\
\hline$t$ & Time \\
\hline$\psi_{* *}(t)$ & Degree of fulfillment of a criterion $* *$ \\
\hline$c_{s u b}$ & Soft upper bound \\
\hline$c_{h l b}$ & Hard lower bound \\
\hline$w_{* *}(t)$ & Weight given to a criterion $* *$ \\
\hline$\zeta(t)$ & Network liveliness \\
\hline$F$ & Level of functionality \\
\hline$L_{F}$ & Network lifetime \\
\hline
\end{tabular}

period of time until the liveliness value reaches 0 , and the network is considered non-functional. All the possible intermediate values are calculated in the same manner. This kind of definition produces lifetime values in the form of time-functionality pairs, as we introduced at the beginning of this section.

This new definition of the network lifetime has several advantages. Firstly, it is able to take the network's application into account in a dynamic way. As a result, it is suited to evaluate controlled degradation strategies. It can also measure the performance of algorithms in different WSN scenarios, allowing for realistic comparisons. This definition also provides WSN developers with a tool to characterize their applications' needs prior to deployment.

\section{B. Controlled degradation scenarios}

This new definition of the network lifetime, and its focus on the application and its level of functionality, allows us to propose the concept of the controlled degradation of the network. As we stated in the introduction, it is very desirable for the user or the application to have control in the way the WSN stops functioning. Generally, this process is not supervised so the different nodes in the network deplete their batteries and die in an almost random manner.

A lot of WSN scenarios would benefit from handling this degradation process, eventually increasing their network lifetime. The actual implementation of this control of the degradation would be intrinsically related to the specific application of the WSN.

For instance, a WSN scenario can have several redundant sensor nodes measuring a certain magnitude. These nodes send their measures to a high-level node that performs some data aggregation and forwards the results to a base station. In such a network, the death of the high-level node is much more pernicious than that of the sensing nodes. Furthermore, it is very probable that this high-level node is the first to die, given that it performs more demanding tasks. However, this final period of the network's operation can be controlled. The WSN can switch to a "degradation mode" that could consist of turning off all redundant sensors and disabling data aggregation at the high-level node. This way, this node could perform its forwarding operation for a much longer time than in the normal mode of functioning. Although the quality of the measurements could diminish, the network would be still providing its intended functionality.

We propose the concept of the "controlled degradation of the network" to refer to all the mechanisms destined to manage the final period of operation of WSNs. Our proposed lifetime definition, expressed in the form of timefunctionality pairs, is perfectly suited to test and enhance algorithms in this particular area.

One drawback of controlling the degradation could be that it demands extra resources from the network to perform its strategies. However, these strategies are designed taking into account the typical restrictions of WSNs. In terms of complexity and implementation, our proposed solutions are perfectly suited to be carried out by low-resources WSNs with little overhead.

To prove the validity of our proposals, a proof of concept is presented in the next section.

\section{PROOF OF CONCEPT}

In this section we provide a valid demonstration of our postulates. In the first place we will show the usefulness and potential of our lifetime definition. Secondly, a basic controlled degradation scheme will be described and tested, making use of this definition. Specifically, it is proven that there is a gain of performance, in terms of lifetime, when the network's degradation is controlled.

The proof of concept is based on a series of simulations done over a Cognitive Wireless Sensor Network (CWSN) simulator [11], based on the well-known Castalia platform [12]. The reason for using a cognitive simulator is that we are planning on adding some cognitive features to our strategies in the future, although they are not currently implemented.

\section{A. Scenario definition}

The simulated scenario has typical characteristics for this field. Its area is a $40 \times 40 \mathrm{~m}$ square and the simulation time is 400 seconds. There are a total of 104 nodes, including a sink (0), four routers (1-4) and 100 packet generators (5-104). The total simulation area is divided into four equal regions. The sink and the routers positions are fixed, the sink in the center of the square, and each router in the center of each region. The packet generators are uniformly deployed, 25 in each region. This deployment can be seen in Fig. 1. All nodes use the radio parameters of the known Texas Instruments CC2420 transceiver. The packet size is 12 bytes, while the packet rate of the generators varies in the different tests. All these values are 


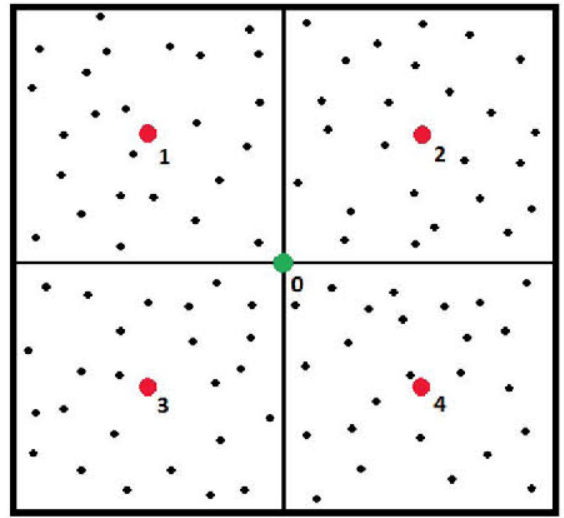

Fig. 1. Node deployment in the simulated scenario.

typical of this field and allow for realistic but not too long simulations.

The generators in each region send their packets to their corresponding router which, in turn, forwards them to the sink. The router nodes only forward received packets to the sink but do not send packets of their own. The sink node only receives packets.

\section{B. Use of the network lifetime definition}

To show the application of our network lifetime definition we have simulated our scenario with the following characteristics. All the packet generators have the same packet rate of 10 packets/s. The sink and the router nodes have an initial energy of $18000 \mathrm{~J}$ (approximately corresponding to two AA batteries). In turn, the generators have a different initial energy depending on their region. The initial energies are 2, 6, 10 or $14 \mathrm{~J}$, for regions 1 to 4 respectively. This causes the generator nodes in the different regions to die gradually, one region at a time (while the sink and the routers are still functioning). This is reflected in the aggregated throughput of received packets measured at the sink, which results in a decreasing function. While this difference in initial energies is not typical of a real scenario, our intention in this section is to showcase the potential and flexibility of our lifetime definition. It is worth noting that the scenario is very realistic on every other aspect so some packets are lost due to noise, collisions, full buffers, etc.

In order to present clear results, we set the aggregated throughput measured at the sink to be the only metric that contributes to the liveliness expression for these simulations. This is done without loss of generality. We have set the soft upper bound, $c_{\text {sub }}$, at 70 packets/s, and the hard lower bound, $c_{h l b}$, at 20 packets/s. The liveliness, $\zeta(t)$, is defined as follows and is represented in Fig. 2:

$$
\zeta(t)=\psi_{t h}(t),\left(w_{t h}=1\right),
$$

with

$$
\psi_{t h}(t)=\left\{\begin{aligned}
0, & \text { if throughput }<20 \\
\frac{\text { throughput }-20}{70-20}, & \text { if } 20<\text { throughput }<70 \\
1, & \text { if throughput }>70
\end{aligned}\right.
$$

In Fig. 2 we have marked the two most interesting lifetime values. Firstly, the leftmost vertical line marks the time where the functionality drops from $100 \%$, corresponding to:

$$
L_{100 \%}=\{121 s, 100 \%\}
$$

The second vertical line corresponds to the total lifetime, marked at the moment the WSN reaches a $0 \%$ of functionality.

$$
L_{0 \%}=\{302 s, 0 \%\}
$$

As expected, the lifetime value for a lower functionality is greater than that of a higher one. It can be seen that being able to measure different lifetime points and representing them as time-functionality pairs is very illustrative.

Another benefit of our definition is the possibility of considering the application requirements in a dynamic manner. This is demonstrated in the following test, based on the same scenario. In Fig. 3 we represent the fact that, from the 100 to the 200 second marks, the application had higher quality requirements and set the bounds to different values, 100 packets/s for $c_{\text {sub }}$ and 40 packets/s for $c_{\text {hlb }}$.

The graphs in this subsection show the usefulness and potential of our definition.

\section{Controlled degradation strategies}

The degradation control strategy that we propose in this work consists of implementing a prioritization scheme. It does not fully exploit the possible ways in which the degradation can be controlled, but it serves as a good proof of concept.

In this case the generator nodes and the sink have an initial energy of $18000 \mathrm{~J}$, so they will not die during the simulation. In turn, all four routers, which will perform the

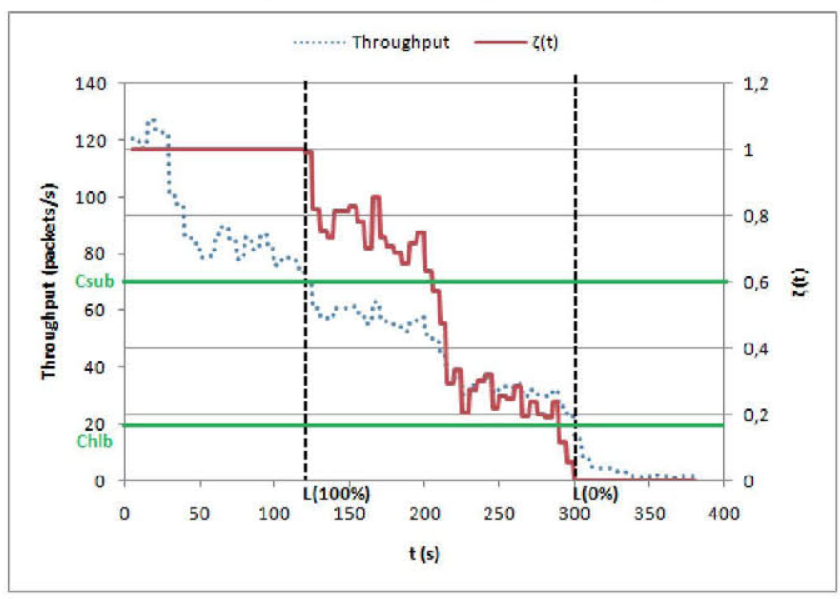

Fig. 2. Liveliness expression and two main lifetime points. 


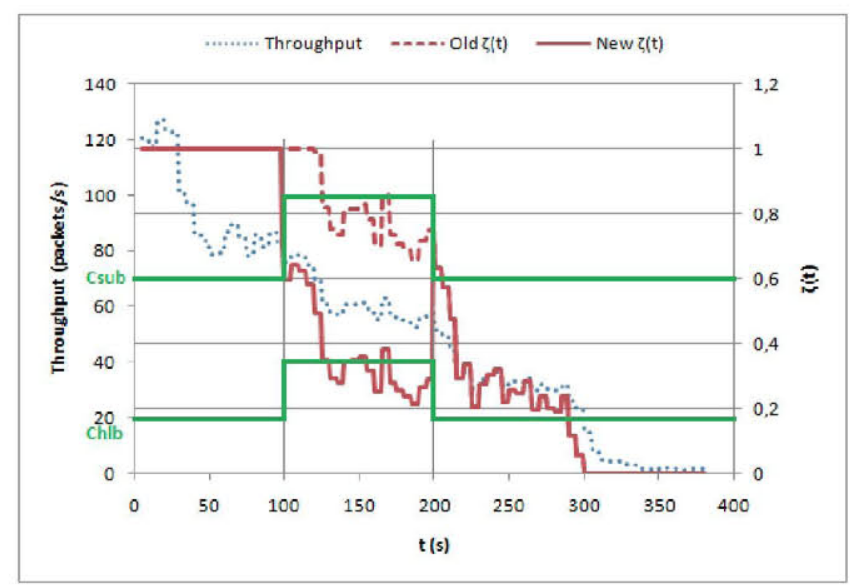

Fig. 3. Liveliness expression with a dynamic adjustment to the application requirements.

controlled degradation strategy, have a limited energy of 6 J. All generators have a packet rate of 2 packets/s. As in the previous case, we link the liveliness expression to the throughput metric measured at the sink, with the following values:

$$
\zeta(t)=\psi_{t h}(t),
$$

with $\psi_{t h}(t)$ being the same expression as the one in section III.A.1).

The controlled degradation strategy, performed by the routers, consists in prioritizing packets based on their source. It assigns a higher priority to some of the generator nodes and enforces this prioritization in the router queues. This is implemented by instantly forwarding prioritized packets while queuing non-priority ones for a certain time. This strategy proves that one can increase the duration of a certain service (in this case the delivery of information from the prioritized sources) at the expense of reducing the lifetime of other less important traffic.

Firstly, we display a reference simulation of the scenario in which the degradation strategy is not performed. This is done in order to have reference values when testing the degradation mode. The bounds $c_{s u b}$ and $c_{h l b}$ are 80 and 20 packets/s, respectively, attending to the throughput values. This is shown in Fig. 4, where the aggregated throughput measured at the sink and its corresponding liveliness are depicted.

The total lifetime, with a functionality of $0 \%$, achieved in this scenario with no controlled degradation is:

$$
L_{0 \%}=\{207 s, 0 \%\}
$$

Now we apply our controlled degradation strategy from the beginning of the simulation. We select 5 generator nodes in each region, a total of 20 , to be prioritized sources. The other 80 generator nodes are non-prioritized. This degradation scheme does not affect the prioritized traffic so the throughput of each individual prioritized node is the

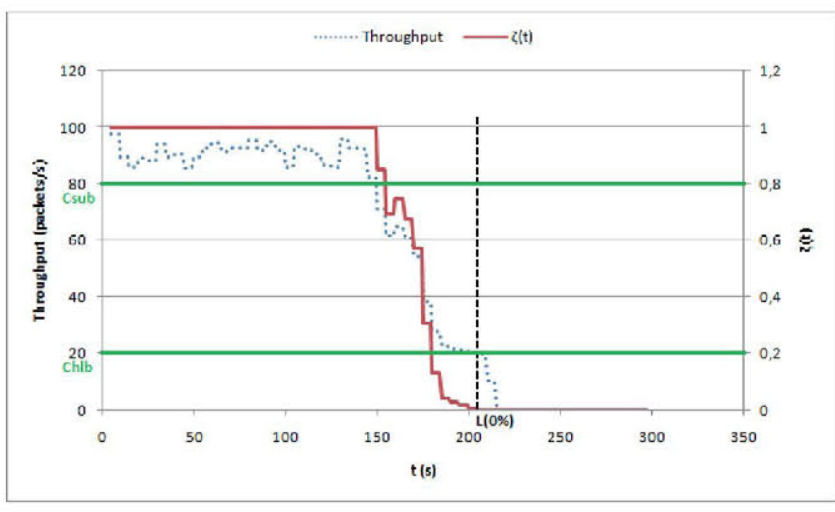

Fig. 4. Liveliness expression with no controlled degradation strategy.

same as in the reference scenario. On the contrary, nonpriority packets are queued and sometimes discarded, resulting in a lower throughput for this traffic. This has the result of reducing the energy consumption and achieving a higher lifetime for the prioritized data flow, without affecting its quality.

Given that only 20 of the total 100 nodes contribute to the prioritized traffic, their corresponding aggregated throughput at the sink is a fifth of the one in the scenario with no controlled degradation. In order to have comparable results, the new bounds for the prioritized flow are a fifth of the previous ones, $c_{\text {sub }}{ }^{\prime}=16$ packets/s and $c_{h l b}{ }^{\prime}=4$ packets/s. In the case of the non-priority traffic these values are decreased attending to its lower throughput, $c_{\text {sub }}{ }^{\prime \prime}=4$ packets/s and $c_{\text {hlb }}{ }^{\prime \prime}=1$ packet $/ \mathrm{s}$. The corresponding liveliness expression is displayed in Fig. 5.

It can be seen that the network has achieved a considerably greater total lifetime:

$$
L_{0 \%}=\{283 s, 0 \%\}
$$

which is an increase of $37 \%$ over the case with no controlled degradation (207 s). This increase in lifetime is accomplished without affecting the quality of the prioritized traffic, but with a considerable decrease in the nonprioritized traffic throughput.

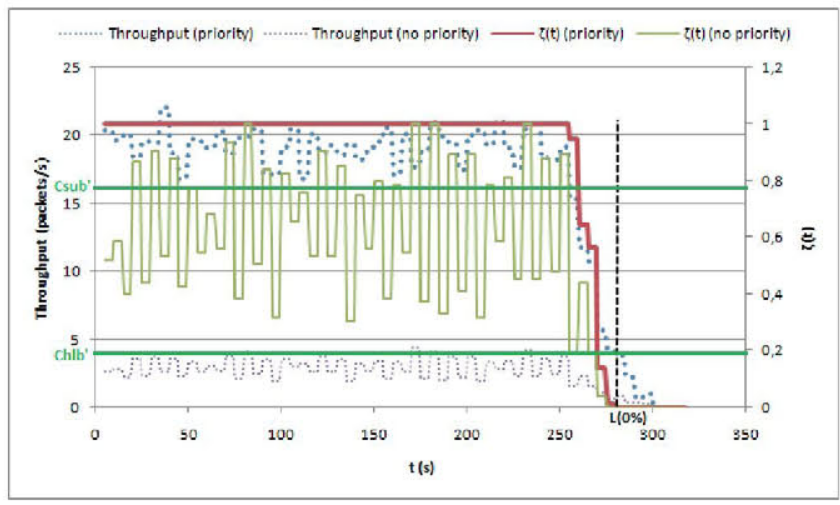

Fig. 5. Liveliness expression applying the controlled degradation strategy. 
This same thing can be done, more realistically, in a unique simulation. With the same scenario and parameters, we start the simulation with no control of the degradation, and at the 100 second mark we enable the prioritization strategy. This is shown in Fig. 6.

This scenario shows the realistic possibility of enabling the degradation mode in the middle of the network's operation. It achieves a total lifetime of:

$$
L_{0 \%}=\{248 s, 0 \%\}
$$

which is a $20 \%$ increase over the case with no control of the degradation.

\section{CONCLUUSIONS}

There is a huge interest in the research community to increase the energy efficiency and, consequently, the lifetime of WSNs.

Usually, WSN developers make use of generic algorithms and communication schemes, without fully considering the specific necessities of their application. We defend that taking the network's purpose into account can provide a great improvement of performance both in terms of lifetime and quality of service.

Specifically, this can be applied to control the behavior of the network when nodes are nearly depleted and resources are scarce, what we propose to include under the concept of the controlled degradation of the network. In this paper, we have demonstrated that this degradation control can effectively increase the network's lifetime.

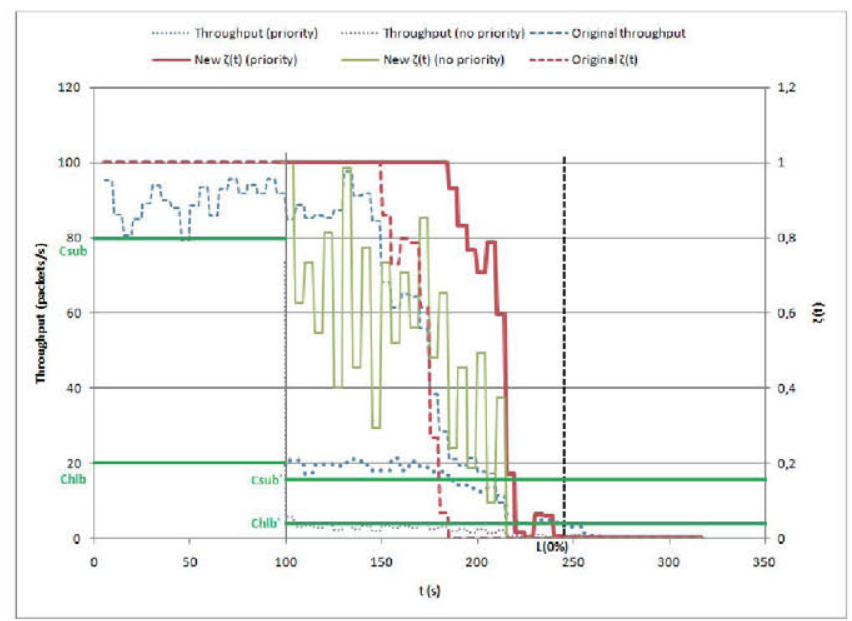

Fig. 6. Liveliness expression where the controlled degradation strategy is applied at second 100 .
The existing network lifetime definitions in the literature are not suited to describe and test our controlled degradation concept. As a result we have introduced a new formal lifetime definition, which is flexible and comprehensive, and is able to take the application's requirements into account in a dynamic way.

\section{ACKNOWLEDGMENT}

This work was funded by the Spanish Ministry of Economy and Competitiveness, under INNPACTO program (Reference grant SETH: IPT-2012-0703-380000) and under RETOS COLABORACION program (Reference grant S4BIM: RTC-2014-2040-7).

\section{REFERENCES}

[1] Cisco, "Cisco Visual Networking Index: Global Mobile Data Traffic Forecast Update, 2014-2019," 2015. [Online]. Available: http://www.cisco.com/c/en/us/solutions/collateral/serviceprovider/visual-networking-index-vni/white paper cll520862.html. [Accessed: 21-April-2015].

[2] T. Rault, A. Bouabdallah, and Y. Challal, "Energy efficiency in wireless sensor networks: A top-down survey," Comput. Networks, vol. 67, pp. 104-122, Jul. 2014.

[3] Y. Chen and Q. Zhao, "On the lifetime of wireless sensor networks," IEEE Commun. Lett., vol. 9, no. 11, pp. 976-978, Nov. 2005.

[4] V. Cherkassky, "A measure of graceful degradation in parallelcomputer systems," IEEE Trans. Reliab., vol. 38, no. 1, pp. 76-81, Apr. 1989

[5] N. Passas and L. Merakos, "A graceful degradation method for congestion control in wireless personal communication networks," in Proceedings of Vehicular Technology Conference - VTC, 1996, vol. 1, pp. 126-130.

[6] I. Kang and R. Poovendran, "Maximizing Network Lifetime of Broadcasting Over Wireless Stationary Ad Hoc Networks," Mob. Networks Appl., vol. 10, no. 6, pp. 879-896, Oct. 2005.

[7] Q. Dong, "Maximizing system lifetime in wireless sensor networks," in IPSN 2005. Fourth International Symposium on Information Processing in Sensor Networks, 2005., 2005, pp. 13-19.

[8] B. Chen, K. Jamieson, H. Balakrishnan, and R. Morris, "Span: An energy-efficient coordination algorithm for topology maintenance in ad hoc wireless networks," Wirel. networks, vol. 8, no. 5, pp. 481494, 2002.

[9] D. M. Blough and P. Santi, "Investigating upper bounds on network lifetime extension for cell-based energy conservation techniques in stationary ad hoc networks," in Proceedings of the 8th annual international conference on Mobile computing and networking MobiCom '02, 2002, p. 183.

[10] I. Dietrich and F. Dressler, "On the lifetime of wireless sensor networks," ACM Trans. Sens. Networks, vol. 5, no. 1, pp. 1-39, Feb. 2009

[11] A. Araujo, E. Romero, J. Blesa, and O. Nieto-Taladriz, "A framework for the design, development and evaluation of Cognitive Wireless Sensor Networks," Int. J. Adv. Telecommun., vol. 5, no. 3 and 4, pp. 141-152, 2012.

[12] Castalia Wireless Sensor Network Simulator website. https://castalia.forge.nicta.com.au/index.php/en/index.html. [Accessed: 21-April-2015]. 